\title{
Design of Smart Universal Remote using Mobile for Home Automation
}

\author{
Joydeep Roy ${ }^{1}$, Joyanta Kumar Roy ${ }^{2}$ \\ ${ }^{I}$ Project Engineer, Wipro Tech, India. \\ ${ }^{2}$ Dean (Research \& Consultancy), Professor Head, Department of Electronics and Communication \\ Engineering, MCKV Institute of Engineering, W.B, India
}

\begin{abstract}
Controlling Home Appliances remotely is à main part of automation. There is a great deal of inconvenience in controlling each digital home appliance with its own separate remote. In this paper we present an advanced universal remote which can control and regulate lights, fans and also any IR controlled Home appliance like TV, home theater, air conditioner etc. This paper gives the design on how to build this remote and how we can turn our smart phone into a true universal remote which will be both versatile and have capability to easily learn new devices and new IR protocols.
\end{abstract}

Keywords: Home Automation, IR decoding, MSP430 Microcontroller, Universal Remote

\section{Introduction}

In our day to day life, we use various gadgets such as television, set top box, air conditioners, home theater, DVD player and many other remote operated devices for comfort and happiness. Different gadgets mean maintaining different remote controls which are not only clumsy but also difficult to manage. Universal remote control simplifies our life because it helps us control any IR devices like TV, air conditioner, home theater including lights and fans. But, because of many technological complexities it becomes difficult to build a single IR based universal remote which can control all available IR based electronic gadgets from different manufacturers.

The rapid growth in mobile communication system in present era is changing people's life and work style. Fast development of mobile technology has brought the world in our grasp. Functionalities of electronic gadgets like computer, IPod, camera etc. are now made available in a single mobile phone. Smart phones are already feature-perfect and can be made to communicate to any other devices in an ad-hoc network with connectivity options like Bluetooth and Wi-Fi. Most of the home appliances use Infrared based controlling systems. Therefore, a simple mobile phone cannot be used directly to control any such appliances. Some special hardware is required to turn a mobile phone into a universal remote capable of controlling the IR based home appliances. Automation of the surrounding environment of a modern human being allows increased work efficiency and comfort. There has been a significant development in the area of an individual's routine tasks and those that can be automated. So, many workers are trying to implement universal remote using smart mobile as a part of Home Automation System.

Panth and Jivani [1] have developed a Home Automation System using mobile in Android platform. The system has been designed to automate 8 bit Bluetooth enabled micro controller, which controls lights and fans using on and off relay switches. But this system lacks the capability of controlling any IR controlled devices.

Mahdi and Alankar in the year 2014 [2] proposed a system for wireless controlling of remote electrical devices using android smart phone. The system uses GPRS technology to turn on and off a LED from any part of the world.

Deepti Shastri [3] proposed to design a mobile based home automation system without operating system constraints. Similar On/Off control application has been made by Puthanial et al. [4] using android and

Bluetooth technology enabled smart phone. Ahsan K, et al. [5] reviewed Unicon Remote Control model - A mobile system for assistive technology and provides the idea of Universal Remote Control application for smart phones with new feature of automatic detection of nearby device and controlling different devices through WiFi or Bluetooth. Patil \& Dixit in their work "Universal Remote for next generation" [6] implemented universal remote control based on Programmable system on chip (PSoc).

Gaurav Chitranshi et al. [8] propose an approach to completely remove the remote and use android mobile with infrared port facility to control the home appliances. Mitul, et al.[9] designed and constructed a Microcontroller based Remote Control which specifically meet the requirement of control of Home appliances through any remote control device that is workable within a room. 
Taewan Kim et al. [7] proposed a PC based interface for end users to use Universal Remote Control (URC) conveniently. The proposed URC can easily connect ubiquitous home automation and security environment with the total solution.

Smart home is a home equipped with special facilities to enable occupants to control or program an array of automated home electronic devices. For example, a home owner on vacation can arm a home security system, control temperature gauges, switch appliances on or off, control lighting, program a home theater or entertainment system, and perform many other tasks. Smart home became smarter if the controlling can be done from any remote place. Shahriyar et al. [10] studied the use of Mobile telephony in remote controlling of home appliance.

In all the above works there are some common missing features. First, there is no or poor self-learning feature. Second, all the proposed methodologies are either sensitive or fixed to a particular set of IR protocols.

The main objectives of this paper are as follows:

(i) The URC should have ability to learn new device quickly- Each button on a remote corresponds to a particular bit pattern. Bit patterns vary from devices to devices. For example, a Power On Key bit pattern will be different for TVs of different makes like Sony and LG. The objective of our paper it to ease the process of learning new bit pattern such that the universal remote can be made compatible with any new devices one brings in home.

(ii) Self-adaptive/ insensitive to underlying IR protocol- IR protocols or format used in various IR remotes vary to a great extent. Currently there are many available IR formats like RC5, NEC, RC6, Sony Code SIRCS, Pansasonic command, Sharp Data format etc. Bit logic for all of these protocols is different. A true Universal remote must not be sensitive to the underlying protocol used. It must be able to control a TV from any manufacturers following any protocol may it be Sony, Panasonic or Sharp. Even if some day a new protocol comes up, the universal remote must be self-adaptive to that change and should work.

(iii) Control Fans and Lights in a room remotely - In addition to IR based devices we have added remote control and regulation of lights and fans from smart phone.

\subsection{General Overview}

\section{System Architecture And Proposed Methodology}

The whole system is divided into the two functional parts. First is the teaching module which required by the URC to learn new devices and their new IR bit patterns. Second part of the system is the operational module. This part comes to play whenever the URC is operated and whenever a user wants to control IR devices or lights and fans from his Mobile phone.

\subsubsection{Teaching Module}

Fig. 1 describes a block diagram of the Teaching module. To teach the system any new function's bit pattern we will be using this circuit. For example, say if we bring in home a new TV and want to add its remote functions to our universal remote, then we need to need to teach our universal remote the IR bit codes of the various functions. This circuit will receive an IR signal from any standard remote, decode it into bit pattern and will send it as microphone input for Mobile recording. The mobile recording should be done in .wav file with PCM encoding. WAV is a Microsoft and IBM audio file format standard for storing an audio bit stream. Encoding format PCM or Pulse Code Modulation is a method to digitally represent sampled analog signal.

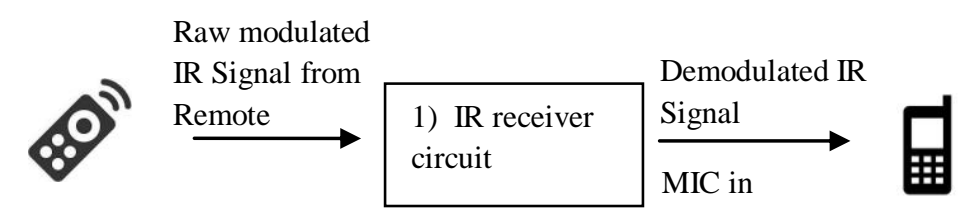

Figure 1: Universal Remote Mobile Learning Process

\subsubsection{Operational Module}

Fig 2. portrays the overview of the operational section of URC. Whenever a user wants to control any IR device from his mobile and presses any button to perform a specific function this part of the system gets activated. The pre-recorded IR bit pattern for that particular function is played back on the mobile phone through the mobile's headphone audio out. 


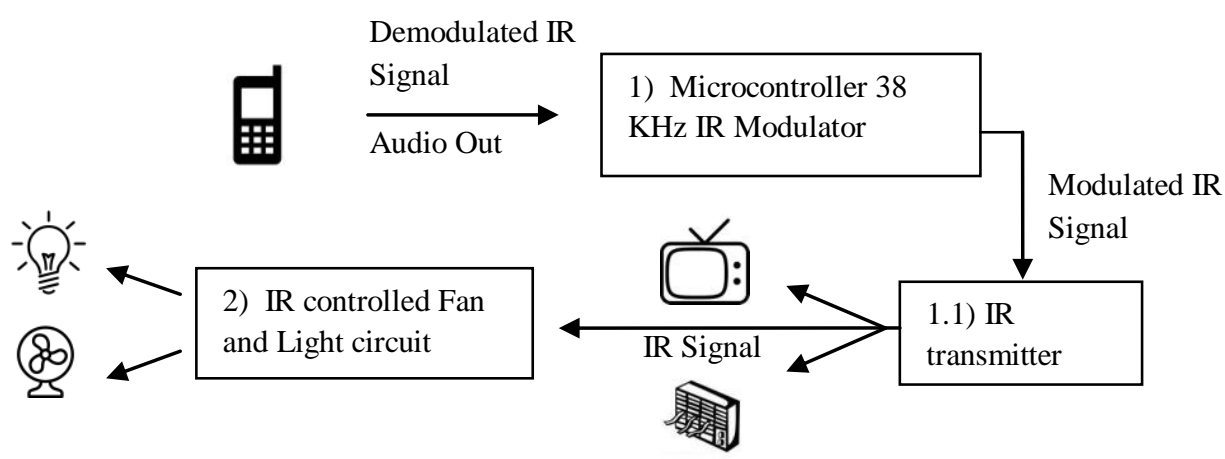

Figure 2: Operational system of mobile as universal remote

A room may have hundreds of tiny IR sources, the lamps around, even the cup of hot coffee. A way to avoid all those other sources remote control pulsates its IR signal at a particular frequency, most common being $38 \mathrm{KHz}$. In our project we are using Microcontroller MSP430G2231 to modulate the IR Bit patterns with a 38 $\mathrm{KHz}$ carrier frequency before sending it out through the IR Transmitter. The microcontroller takes recorded IR waves (demodulated) as input and generates IR wave with $38 \mathrm{KHz}$ modulation. The modulated IR wave is then amplified and sent out through IR transmitter. Fig. 3 shows the input demodulated IR signal marked by 1 and the output modulated IR signal marked by 0 .

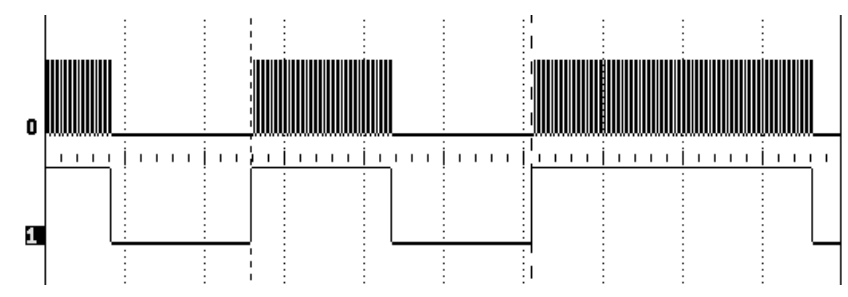

Figure 3: Operational system of mobile as universal remote

The second part of this operational module is IR controlled Fan and Light Circuit. IR signal received from the IR transmitter is decoded by an IR Receiver. The decoded IR signal passes through a SIS-2 module for analysis. If the bit pattern matches the pre -stored bit pattern then relay switch are turned on and off. The bit patterns for turning on and off fan, fan speed up, fan speed down, turning lights on and off are all preprogrammed in the system. Microcontroller MSP430G2231 is used to control fan speed by generating triggering pulse to the Triac controlling phase shift.

\subsection{Hardware Design}

\subsubsection{IR Receiver Circuit}

To build the IR Receiver we have used IR Receiver TSOP34156. [12] It is a simple IR receiver for infrared remote control which has low power consumption and is easy to use. This receiver is also compatible with all common IR remote control data format. The receiver receives an IR signal and demodulates it. Fig. 3 shows the IR receiver diode and its associated circuitry. The demodulated IR wave from Pin PD.0 is to be connected to microphone in for IR bit pattern recording.

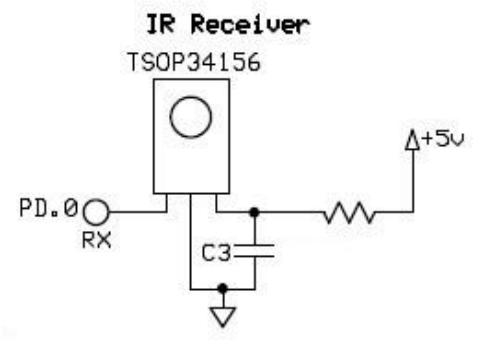

Figure 3: IR Receiver circuit 


\subsubsection{IR Modulator and Transmitter Circuit}

The demodulated IR signal is passed as input at Pin 2 of $\mathrm{C} 1$ capacitor as shown in Fig 4. The signal recorded in mobile is of low amplitude and can't be properly detected by the microcontroller. So, we first amplify it using an OPAMP (U1) and then pass it as input to MSP430G2231 at Pin 1.9 as shown in Fig. 4.

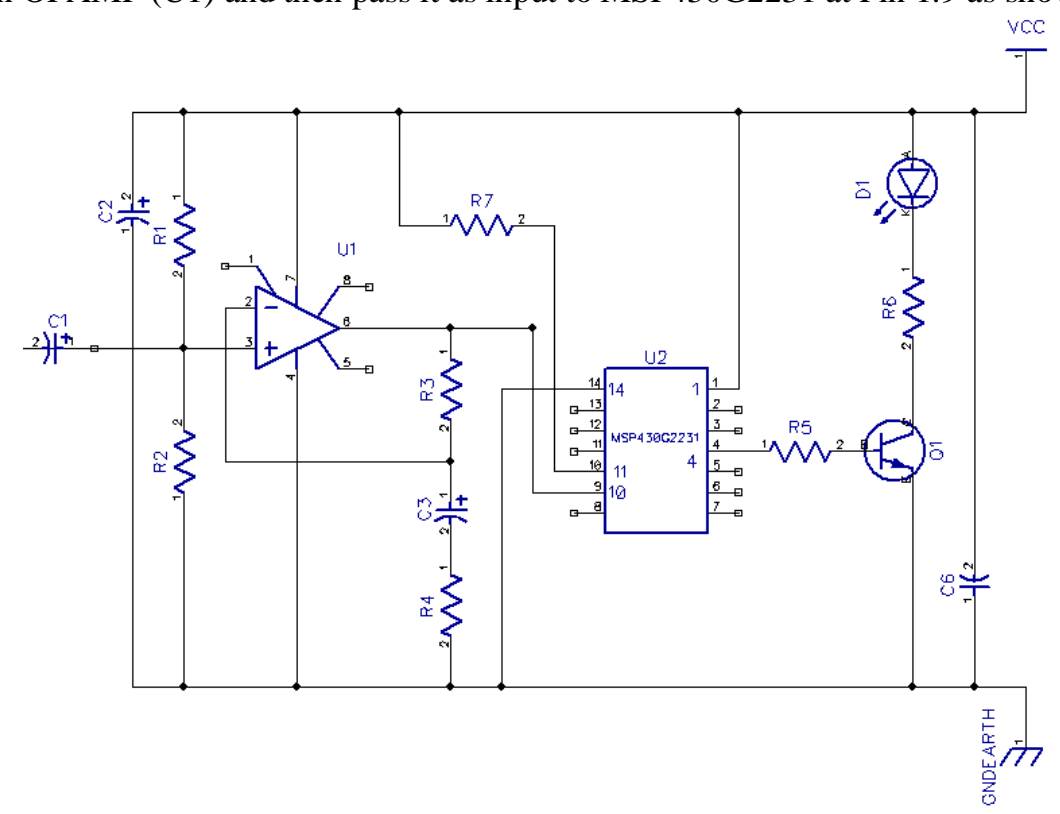

Figure 4: IR Amplifier, Modulator and Transmitter circuit

MSP430G2231 has been used [11] for modulation. MSP430G2231 is an ultra-low power controlled micro controller featuring various features targeting multifarious applications. The architecture combines with five low power modes and features a powerful 16 bit RISC CPU, 16 bit registers, and constant generators that contribute to maximum code efficiency. The digitally controlled oscillator (DCO) allows wake up from low power modes to active mode in less than $1 \mu \mathrm{s}$. It has inbuilt 16-bit timer with two capture/compare registers. It also has a 10-Bit $200 \mathrm{ksps}$ A/D converter with internal reference, sample-and-hold, and auto scan features.

MSP4302231 has built in serial on board programming capabilities. Before we place the microcontroller in the circuit shown in Fig 4, we need to program it such that it takes demodulated IR Signals as input (P1.9) and gives IR signals modulated $38 \mathrm{KHz}$ in output (Pin P1.4). To program the MSP430G2231 we used MSP430 Launch Pad [14] which is a microcontroller development kit from Texas Instruments. The MSP430 Launchpad has an onboard flash emulator, USB, 2 programmable LEDs, and 1 programmable push button. Once connected to computer via USB we can use IDE like Code Composer Studio to code and burn the programs in the micro controller. Fig. 5 illustrates the flowchart of IR signal modulation using MSP430G2231. The codes are written in Microcontroller $\mathrm{C}$ programming language 


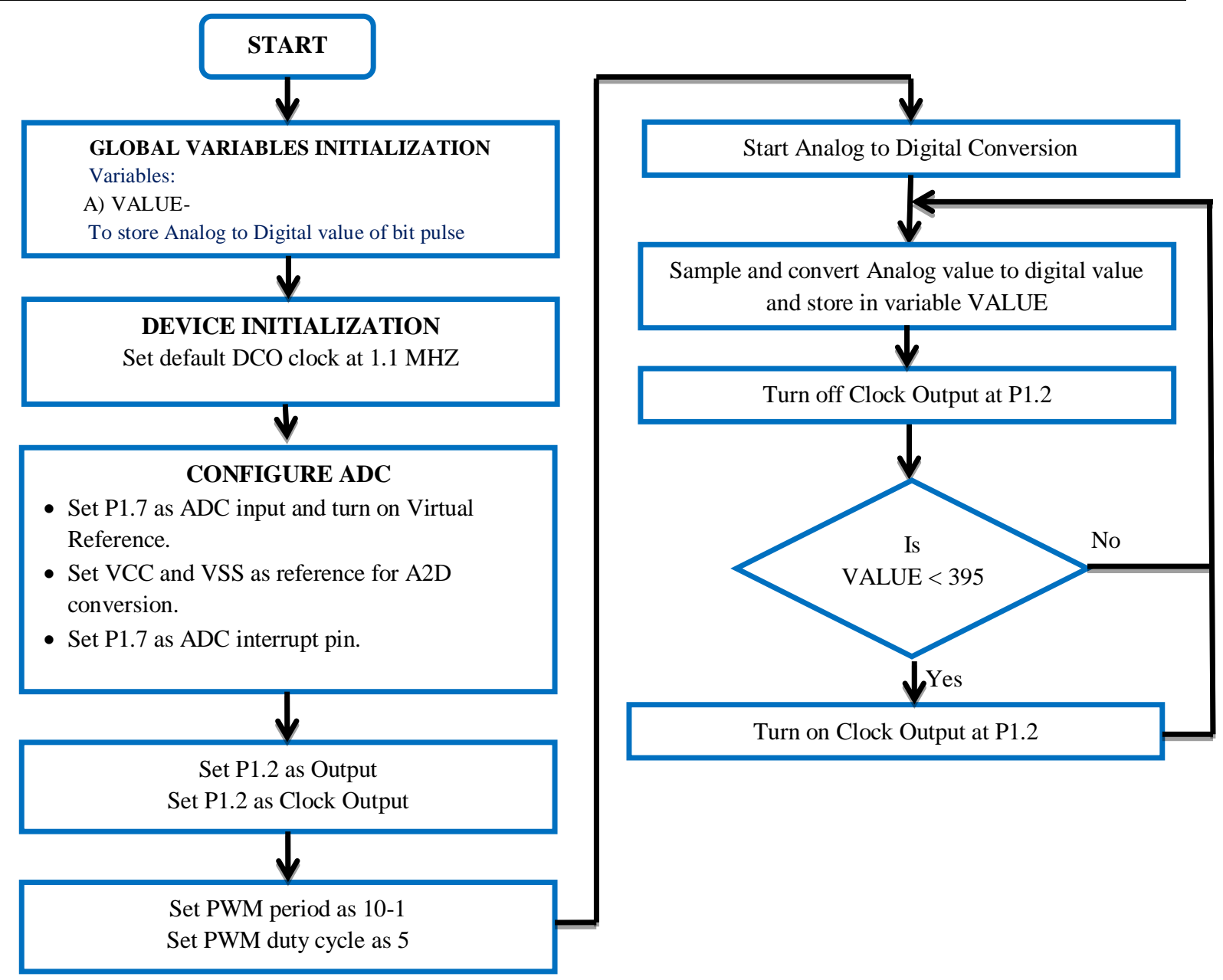

Note: The IR signals get recorded in inverted form on mobile. Analog to Digital Converted Value < 395

Figure 5: Program Flowchart for modulating is experimentally found to be 0 logic level.

Once modulation of the IR waves is complete, it needs to be transmitted. To increase the power of the transmitted IR signals we use here a transistor based IR LED driver.

\subsubsection{IR controlled Fan and Light Circuit}

\subsubsection{IR control circuit for Fan}

Fig 6 is the complete circuit diagram for an IR remote controlled Fan regulator. The IR signal is received by IR Receiver diode at pin marked as IR Input in Fig 6. It is then passed to a programmable IR Receiver SIS-2 (labeled as SIS1 in Fig. 6). [13] The SIS-2 monitors an IR receiver, and when a certain remote code is detected, an output pin is toggled. Two outputs are available and any remote IR code can be taught to the SIS-2. This makes it simpler to build an Infrared remote switching solution for a variety of applications. In our project to control fan speed we have pre-programmed the SIS-2 to 2 different IR codes, one to increase fan speed and other to decrease fan speed. IR codes are stored in non-volatile, re programmable memory. SIS-2 has two normal operation modes, toggle mode or High-Low mode. The mode is controlled by Pin 7 of SIS-2 module. If Pin 7 is set to VSS then toggle mode is activated, that is if IR bit pattern matches, a pulse is generated at the designated output pin. If Pin is set to VDD then mode low/high is activated. In this mode the outputs are by default set to low, and it turns and remains high as long as the IR code passed matches. For Fan control circuit we are using High/Low mode in SIS-2. So when the IR bit code of Speed Up signal is received an interrupt is generated at O2 pin of SIS-2 module and it propagates to P1.7 of MSP430G2231. Similarly when IR bit code of Speed down is received, O1 pin generates an interrupt which propagates to P1.4 of microcontroller. 


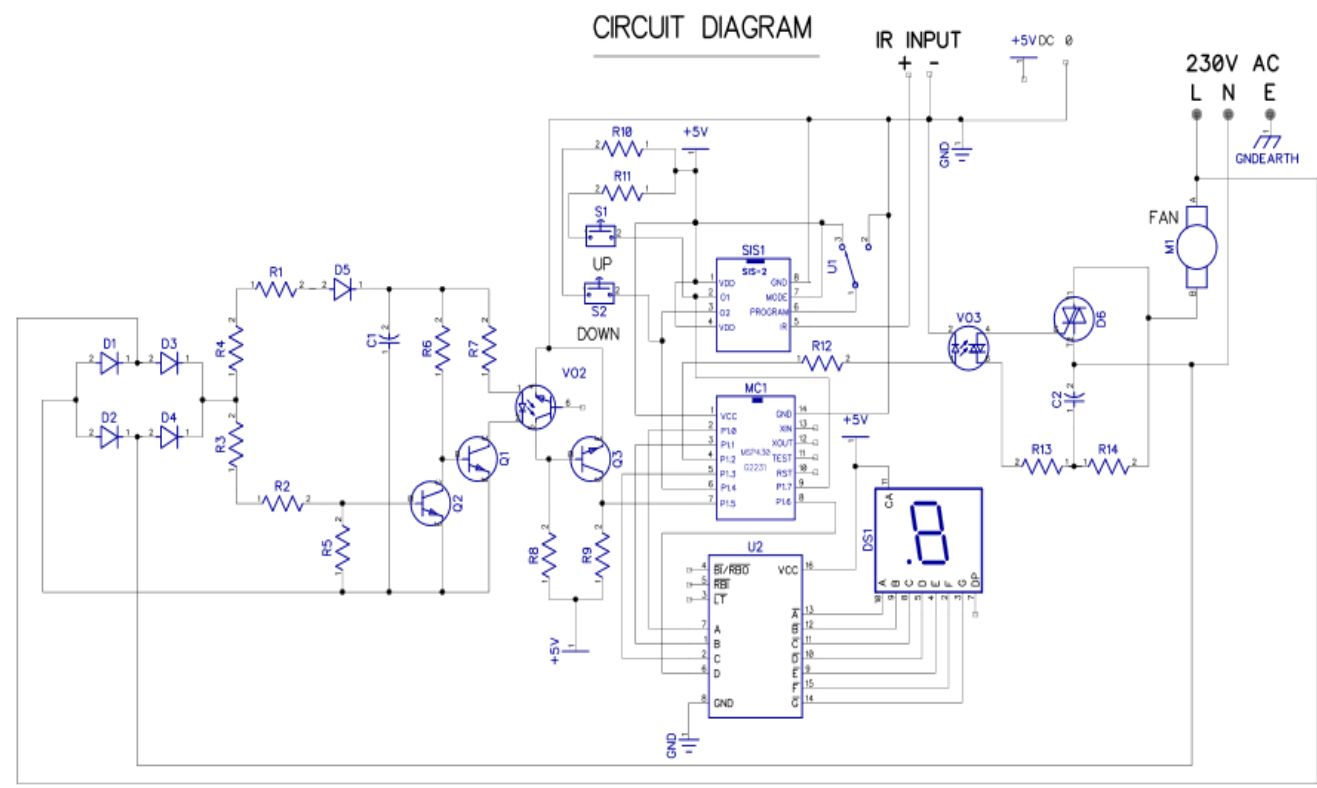

Figure 6: IR Controlled Fan regulator circuit

Left part of the circuit in Figure [6] shows a zero cross detector circuit. Output zero cross pulse is set as input to Pin P1.5 of microcontroller. Microcontroller MSP430G2231 here is programmed to perform 2 main functions. First, it drives the LED display driver chip to display fan speed sequence. Pin P1.0, P1.1, P1.3, P1.6 are set to high or low as per the value of speed step. Second it take the zero cross pulse as input and generates a phase shifted pulse. The degree of phase shift is decided by the speed set by fan speed up and down. This

ultimately drives the gate of opto-isolated Triac connected with the Fan as driver. The speed of the fan can be controlled in 9 steps from low to high. Fig 7 shows a simplified flowchart of the embedded microcontroller program.

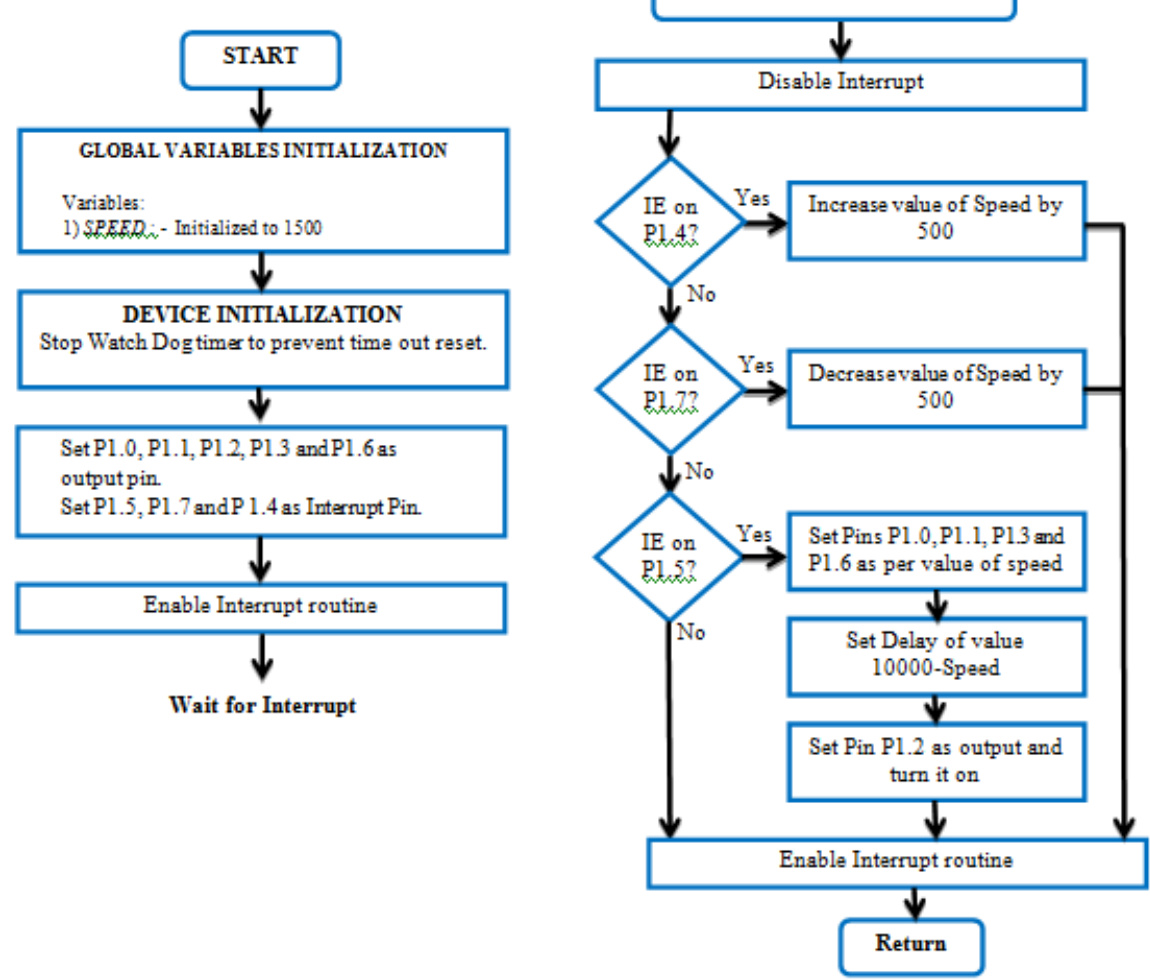

Figure 7: Program Flowchart for fan regulator and LED display driver 


\subsubsection{IR control circuit for Light}

Current project has provisions to control 4 lights remotely. To control 4 lights we are using 2 SIS-2 modules. Each SIS-2 module is pre-programmed with 2 unique IR bit patterns. The IR signals once received are passed on to the SIS-2 module. For light control we are using Toggle modes, i.e. Pin 7 of the SIS-2 modules are connected to Ground. If a bit pattern matches then SIS-2 generates a toggle pulse which operates on a solid state

relay to turn on or turn off the lights. Figure 8 describes the complete circuit built to control lights remotely through IR.

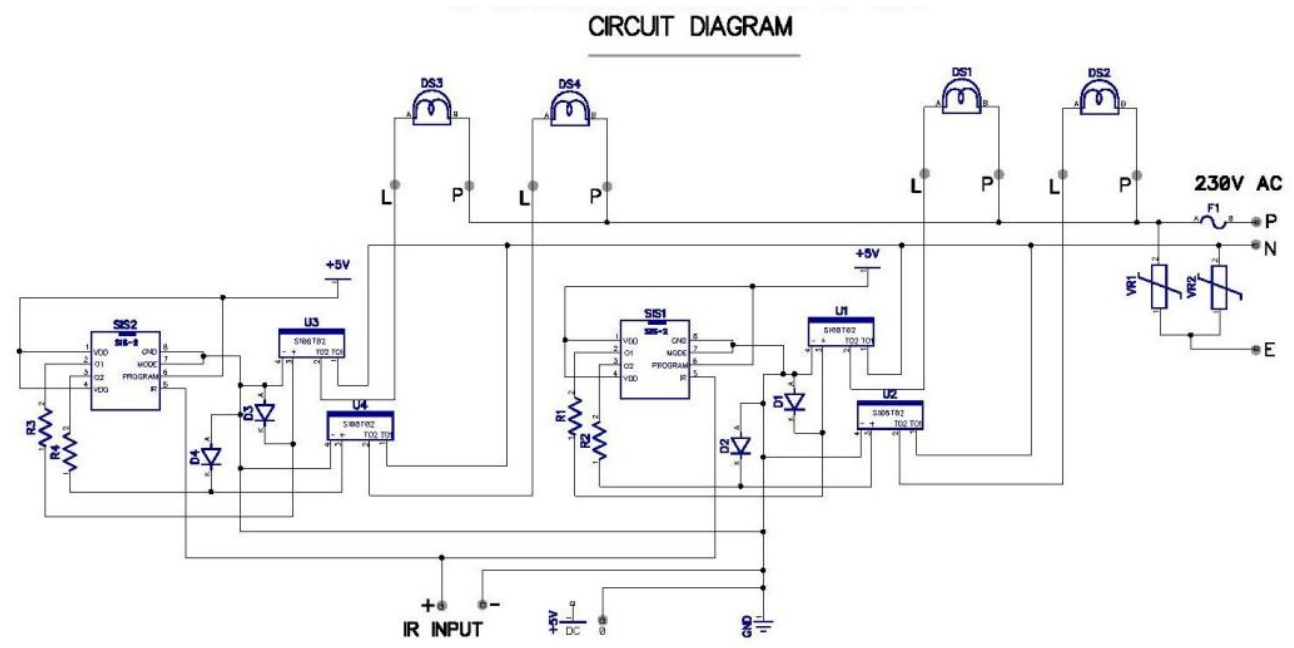

Figure 8: IR Controlled Lights circuit

\section{Results and Discussion}

The prototype of Universal remote was constructed and was found to be working with satisfactory performance. Current prototype has capabilities of 4 lights and 1 fan; however one can easily increase the number of lights and fans to be controlled by simply repeating the circuits currently used. The speed of fan is controlled in 9 steps from zero to full, voltage swings from $0-210 \mathrm{~V}$ AC in output when $230 \mathrm{~V} 50 \mathrm{~Hz}$ is given as input supply. Voltage across light is found to be $215 \mathrm{~V}$ AC when it's switched on. No flickering of light has been observed. Phase control system in Power circuit generated harmonics, so special has been taken in the design to reduce injection of Harmonics in to the power line.

The main feature of the work is the unique way in which the URC learns new devices and functions irrespective of make and manufactures and their underlying IR Protocols. We compared our results with a commercially available mobile based Universal remote control named Beacon by Griffin Technologies. Though Beacon worked well with TV, set top box and DVD/CD/Blu-ray players, it failed to learn new devices like Air conditioners and home theaters. The problem found was the underlying IR protocol used in those IR devices. Our system as planned was found to be self-adaptive and worked not only for TV, set top box and DVD/CD/Blu-ray players but also it worked for air conditioners and home theaters. It was also quick in learning new functions of Air conditioner remote, home theater and satellite TV box.

The future work to be done would be to add wireless capabilities. Instead of using the audio and microphone jack in mobile phone we can use Bluetooth to IR converter to control the IR devices. Next step would be the development of the smart mobile applications in Android platform. The design of the system can be made very user friendly and lots of features to easily manage all devices can be added. The future scopes of this system are endless. The system can also be tweaked to needs of elderly people as and when needed to provide hazard free service.

\section{References}

[1]. Sharon Panth \& Mahesh Jivani, "Home Automation System (HAS) using Android for Mobile Phone", International Journal of Electronics and Computer Science Engineering, 3 (1), 2014, 1-10

[2]. Omar Abdulraheem Mahdi \& Bhavya Alankar, "Wireless Controlling Of Remote Electrical Device Using Android Smartphone", IOSR Journal of Computer Engineering (IOSR-JCE), 16 (3),2014,23-27, e-ISSN: 2278-0661

[3]. Deepti Shastri, "Home Automation System with Universally used Mobile Application Platform", IOSR Journal of Electronics and Communication Engineering (IOSR-JECE), 9 (2),2014, 01-06, e-ISSN: 2278-2834

[4]. M. Puthanial, S. Rajeshwari, Dr. P.C. Kishore Raja \& Dr. P. Shankar, “Android and Bluetooth Technology Enabled 
[5]. Remote Control Using Smart Phone", International Journal of Advanced Research in Electrical, Electronics and Instrumentation Engineering (IJAREEIE), 3 (5), 2014, 9373-9380, e-ISSN: 2278 - 8875

[6]. Ahsan K., Iqbal S., Nadeem A. and Sarim M., "UniCon Remote Control Model - A Mobile System for Assistive Technology", Research Journal of Recent Sciences, Vol. 3(4), 2014, 95-102, ISSN 2277-2502

[7]. Pallavi Patil, M. A. Dixit, "Universal Remote for Next Generation", Proceedings of SARC-IRAJ International Conference, 16th June 2013, Pune, India, ISBN: 978-81-927147-8-3

[8]. Taewan Kim, Kyung Hee, Yongin, Hakjoon Lee \& Yunmo Chung, "Advanced universal remote controller for home automation and security”, IEEE Transactions on Consumer Electronics, 56 (4), 2010, 2537 - 2542, ISSN :0098-306

[9]. Gaurav Chitranshi, Madhvi Gaur, "Using Android Mobile as a Universal Remote Control", International Journal of Engineering Research and Development, 4 (6), 2012, 89-92, e-ISSN: 2278-067X

[10]. Abu Farzan Mitul, Fida Hasan Md. Rafi, Md. Manirul Islam, Mohiuddin Ahmad, "Microcontroller Based Remote Control of Home Appliances", Proceeding of the International Conference on Electrical, Computer and Telecommunication Engineering, 01-02 December 2012 (ICECTE2012), RUET, Rajshahi-6204, Bangladesh

[11]. Rifat Shahriyar, Enamul Hoque, S.M. Sohan, Iftekhar Naim, Md. Mostafa Akbar \& Masud Karim Khan, "Remote Controlling of Home Appliances using Mobile Telephony”, International Journal of Smart Home, 2(3), 2008

[12]. MSP430G2x21, MSP430G2x31 Mixed Signal Microcontroller (Rev. http://www.ti.com/product/msp430g2231

[13]. IR Receiver Diode - TSOP38238, Spark Fun, https://www.sparkfun.com/products/10266

[14]. Programmable IR Receiver - SIS-2, Spark Fun, https://www.sparkfun.com/products/8753

[15]. MSP430 Launch Pad Value Line Development kit, Texas Instruments, http://www.ti.com/tool/msp-exp430g2 